TITLE:

\title{
Localized picosecond-scale process in glassy poly(methyl methacrylate) far below T-g
}

\section{$\operatorname{AUTHOR}(\mathrm{S})$ :}

Kanaya, T; Tsukushi, I; Kaji, K; Gabrys, BJ;

Bennington, SA; Furuya, $\mathrm{H}$

\section{CITATION:}

Kanaya, T ...[et al]. Localized picosecond-scale process in glassy poly(methyl methacrylate) far below T-g. PHYSICAL REVIEW B 2001, 64(14): 144202.

\section{ISSUE DATE:}

2001-10-01

URL:

http://hdl.handle.net/2433/39850

RIGHT:

Copyright 2001 American Physical Society 


\title{
Localized picosecond-scale process in glassy poly(methyl methacrylate) far below $\boldsymbol{T}_{\mathrm{g}}$
}

\author{
Toshiji Kanaya, ${ }^{1, *}$ Itaru Tsukushi, ${ }^{1,2}$ Keisuke Kaji, ${ }^{1}$ Barbara J. Gabrys, ${ }^{1,3}$ Steve M. Bennington, ${ }^{1,4}$ and Hidemine Furuya ${ }^{1,5}$ \\ ${ }^{1}$ Institute for Chemical Research, Kyoto University, Uji, Kyoto-fu 611-0011, Japan \\ ${ }^{2}$ Chiba Institute of Technology, Narashino, Chiba-ken 275-0023, Japan \\ ${ }^{3}$ Faculty of Mathematics and Computing, The Open University in the South, Foxcombe Hall, Boars Hill, Oxford OX1 5HR, \\ United Kingdom \\ ${ }^{4}$ ISIS facility, Rutherford Appleton Laboratory, Chilton, Didcot Oxon, OX11 OQX, United Kingdom \\ ${ }^{5}$ Department of Organic and Polymeric Materials, Tokyo Institute of Technology, 2-12-1 Ookayama, Meguro-ku, Tokyo 152-8552, Japan
}

(Received 22 September 2000; revised manuscript received 27 April 2001; published 17 September 2001)

\begin{abstract}
We carried out incoherent and coherent inelastic neutron scattering on glassy poly(methyl methacrylate) in a wide scattering vector range up to $4.9 \AA^{-1}$ with a high-energy resolution of $0.16 \mathrm{meV}$ to investigate low-energy modes: the so-called boson peak and the picosecond-scale process. First, we studied the basic features of the two modes in the incoherent measurements and found that they are similar to those reported for many glass-forming materials. In the coherent scattering measurements, focusing on the $Q$ dependence of the inelastic scattering intensity, we found that the boson peak and picosecond-scale process modes do not move in phase at a length scale of $\sim 7 \AA$, while the motional coherency is preserved between neighboring side chains in a polymer. These observations suggest that the boson peak and picosecond-scale process are localized modes on two monomers within a polymer chain. The intrachain localization becomes more pronounced with increasing the excitation energy.
\end{abstract}

DOI: 10.1103/PhysRevB.64.144202

\section{INTRODUCTION}

There are still many unsolved problems in the field of the low-energy modes of glasses and supercooled liquids despite the vast body of data collected in the last two decades. ${ }^{1,2}$ One of the most controversial problems is the origin of the boson peak and the picosecond fast process observed in many glass-forming materials in inelastic neutron scattering and Raman scattering spectra. A broad boson peak is universally observed at 1.5-4 meV in glasses well below the glass transition temperature $T_{g}$. When the inelastic scattering intensity is scaled by the Bose factor, the harmonic nature of the scattering becomes evident. The soft potential model, ${ }^{3}$ which postulates a continuous crossover from low-barrier tunneling modes to additional vibrational modes, predicted that the boson peak is a localized mode on several tens to hundreds of atoms. This hypothesis is supported by numerical simulations. ${ }^{4,5}$ Moreover, recent inelastic $\mathrm{x}$-ray scattering (IXS) studies ${ }^{6,7}$ have shown that there is a propagating mode in the low-energy region in glasses. It was originally proposed that the boson peak is a propagating mode: however, the present understanding is that the boson peak is a localized mode which coexists with the propagating mode. The coexistence of the localized and propagating modes is visible in the non-Gaussian behavior of the Debye-Waller factor ${ }^{8-10}$ in a scattering vector $Q$ range larger than about $3 \AA^{-1}$. Hence quantitative evidence slowly emerges that the boson peak is a localized mode.

With increasing temperature, a picosecond-scale process appears in an energy region below the boson peak, so that the spectral shape changes from inelastic to quasielastic like. The scattering intensity of the picosecond-scale process exceeds values expected from the Bose factor due to the anharmonic nature. The onset of the picosecond-scale process is at around $T_{g}$ in several main chain polymers with no long side chains, ${ }^{11-13}$ while it is far below $T_{g}$ in some polymers with
PACS number(s): 61.12.Ex, 61.41.+e, 61.43.-j, 63.50.+x

side chains and/or internal degrees of freedom. ${ }^{14-16}$ The spectral width deduced from Lorentzian fits is almost independent of temperature and the length of scattering vector $Q .^{11,12,17,18}$ The latter, usually interpreted as the picosecondscale process, is a localized mode. However, recent coherent neutron-scattering studies on the fast process in deuterated polybutadiene (PB- $d_{4}$ ) (Ref. 19) have surprisingly provided some evidence that the picosecond-scale process of PB- $d_{4}$ is a damping mode of coherent phonons.

In this work we have performed incoherent and coherent inelastic neutron-scattering measurements on protonated and deuterated poly(methyl methacrylate), respectively. On the basis of the coherent results, we will discuss the spatial scale and the motional coherency of the boson peak as well as the picosecond-scale process in poly(methyl methacrylate).

\section{EXPERIMENT}

Protonated and deuterated atactic poly(methyl methacrylate) (PMMA and PMMA- $d_{8}$ ) which were used for the scattering experiments were prepared using a radical polymerization method. The weight-average molecular weights $M_{w}$ of PMMA and PMMA- $d_{8}$ are 300000 and 320000 , and the polydispersities in terms of $M_{w} / M_{n}$ are 2.0 and 1.9, respectively ( $M_{n}$ being the number-average molecular weight). The glass transition temperatures of both PMMA and PMMA- $d_{8}$ are $104^{\circ} \mathrm{C}(=377 \mathrm{~K})$ as determined by differential scanning calorimetry. The sample thicknesses were controlled in order to give more than $90 \%$ transmission of incident neutrons. The observed scattering laws $S(Q, \Delta E)$ ( $\Delta E$ being the energy transfer of neutron) of PMMA and PMMA- $d_{8}$ can be regarded as incoherent and coherent scattering laws, respectively, because of a very large incoherent scattering cross section of the $\mathrm{H}$ atom and very small ones of $\mathrm{C}, \mathrm{O}$, and $\mathrm{D}$ atoms. ${ }^{20}$ It was reported ${ }^{21,22}$ that the coherent scattering from protonated PMMA at around $Q=1 \AA^{-1}$ is about $25 \%$ of the 
incoherent one. In this work, however, we did not evaluate the coherent contribution in PMMA since the emphasis is on the results obtained for deuterated PMMA.

Elastic and inelastic neutron-scattering measurements were performed on a direct geometry spectrometer MARI installed at a pulsed neutron source in ISIS, Rutherford Appleton Laboratory, Didcot. This spectrometer is designed to have a high-energy resolution of typically $1 \%$ of the incident neutron energy. In the present measurements, we have selected an incident energy of $15 \mathrm{meV}$ to achieve a highenergy resolution $\delta E$ of $0.16 \mathrm{meV}$, which was evaluated from the half-width at half-maximum of the elastic scattering on the neutron energy gain side. The neutron detector banks of the MARI spectrometer are spread from $3^{\circ}$ to $135^{\circ}$ with 1000 detectors, so that a very wide and continuous range of $Q$ is covered. Under the present experimental condition, the $Q$ range was from 0.19 to $4.93 \AA^{-1}$, which is extremely wide compared with a conventional time-of-flight (TOF) spectrometer at a cold neutron source. ${ }^{23}$ This wide $Q$ range is a distinct feature of this experiment. The measurements were performed at 22, 41, 100, 200, and $300 \mathrm{~K}$.

\section{RESULTS AND DISCUSSION}

\section{A. Incoherent scattering}

In order to confirm the basic features of the boson peak and the picosecond-scale process in PMMA, we have carried out incoherent inelastic neutron-scattering measurements far below the glass transition temperature $T_{g}(=377 \mathrm{~K})$ at the above temperatures. The observed incoherent dynamic scattering laws $S(Q, \Delta E)$ were scaled to a reference temperature $T_{\text {ref }}(=100 \mathrm{~K})$ by the Bose factor after correcting for the Debye-Waller factor, which was evaluated from the $Q$ dependence of the incoherent inelastic scattering intensity. The results are shown in Fig. 1. At low temperatures of 41 and 100 $\mathrm{K}$, the dynamic scattering laws $S(Q, \Delta E)$ show a broad excitation peak at $\sim 2 \mathrm{meV}$, which is the so-called boson peak, and the intensities are well scaled by the Bose factor, suggesting the harmonic nature of the excitation. Similar results were reported for many glass-forming materials. ${ }^{18,24-26}$ On the other hand, with increasing temperature, $S(Q, \Delta E)$ cannot be scaled by the Bose factor anymore and shows anharmonic excess inelastic scattering intensity in an energy range below about $4 \mathrm{meV}$. Then, the spectral shape becomes quasielastic like from inelastic like. This excess scattering is the so-called picosecond-scale process. The dynamic scattering law $S(Q, \Delta E)$ of the picosecond-scale process or the excess scattering was fitted to a single Lorentzian function convoluted with the resolution function of the spectrometer. The results of fits are shown by dashed lines in Fig. 1. The agreement between the observed and calculated spectra is good, implying that the single Lorentzian can approximately describe the dynamic scattering law $S(Q, \Delta E)$ of the fast process. The half-width at half-maximum of the Lorentzian is almost independent of temperature and scattering vector $Q$, being about $1 \mathrm{meV}$. Similar results were reported for some glass-forming materials. ${ }^{12,13,15-17,27}$

According to the onset of the picosecond-scale process, the temperature dependence of the mean-square displace-

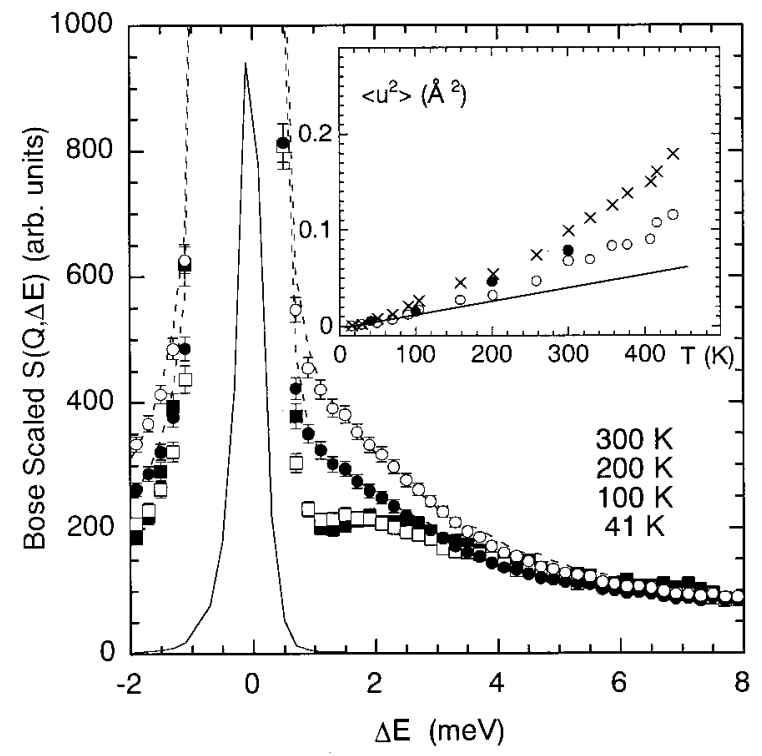

FIG. 1. Incoherent dynamic scattering laws $S(Q, \Delta E)$ of PMMA at $Q=2.48 \AA^{-1}$ scaled to a reference temperature (=100 K) by the Bose factor after correcting for the Debye-Waller factor evaluated from the $Q$ dependence of the incoherent inelastic scattering intensity. The inset shows the temperature dependence of the meansquare displacement $\left\langle u^{2}\right\rangle$ evaluated from the $Q$ dependence of the incoherent elastic scattering with various energy resolution $\delta E$. (×) $\delta E=0.08 \mathrm{meV}$ (Ref. 28), (O) $\delta E=2 \mathrm{meV}$ (Ref. 28), and (๑) $\delta E=0.16 \mathrm{meV}$ (this work).

ment $\left\langle u^{2}\right\rangle$ deviates from the harmonic behavior $\left[\left\langle u^{2}\right\rangle \sim T\right]$ and shows an excess value as shown in the inset of Fig. 1. Also, in this figure the reported values of $\left\langle u^{2}\right\rangle$ are reproduced $^{28}$ to show the anharmonic deviation of the present data. In contrast to the main chain polymers such as polybutadiene, ${ }^{11,12,29}$ the picosecond-scale process present in PMMA is observed far below the glass transition temperature. This low-temperature onset $(\sim 160 \mathrm{~K})$ must be due to internal degrees of freedoms which are not directly related to the glass transition.

In the present measurements, we have extended the $Q$ range up to $4.9 \AA^{-1}$ for the first time, keeping the highenergy resolution $\delta E$ of $0.16 \mathrm{meV}$. Figure 2 shows the scattering laws $S(Q, \Delta E)$ of PMMA obtained at $200 \mathrm{~K}$ for various $Q$ values. The spectra are corrected for the Debye-Waller factor evaluated from the $Q$ dependence of the incoherent elastic scattering intensity. At $Q$ values below $3 \AA^{-1}$ accessible by conventional TOF spectrometers, the observed spectra resemble quasielastic ones, meaning that the picosecondscale process dominates in the low- $Q$ range. At a higher $Q$ value of $4.8 \AA^{-1}$, on the other hand, the boson peak is still observed even above the onset temperature of the picosecond-scale process. The boson peak dominates in the $Q$ range above $\sim 4 \AA^{-1}$. These results have never been reported as far as we know. It is interesting but not easy to answer what this result means. One possible explanation is that the boson peak is a more localized mode than the picosecond-scale process and dominant in the higher- $Q$ range. Another possibility is that boson peak is hidden in the 


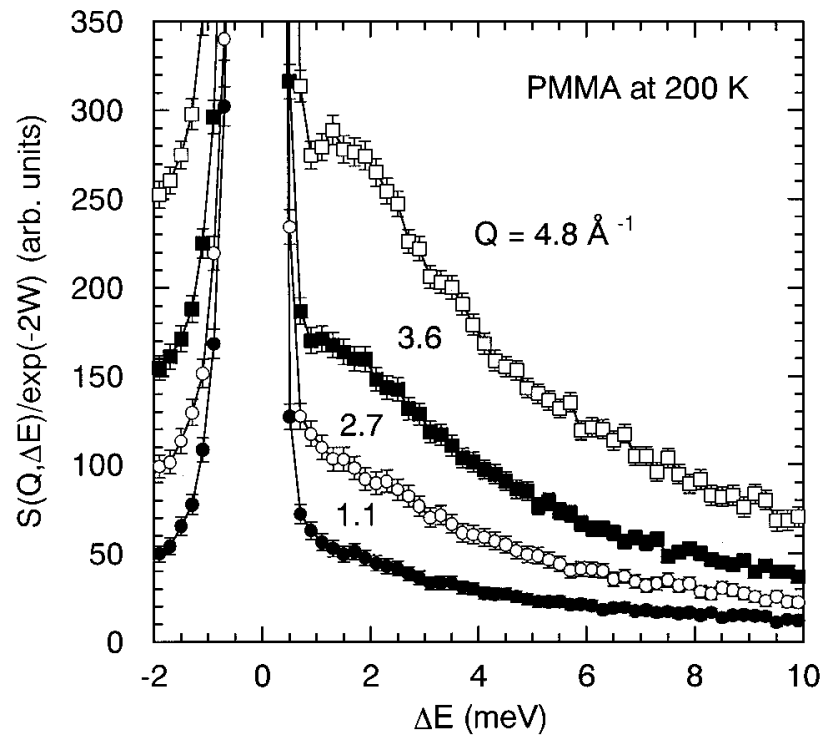

FIG. 2. Incoherent dynamic scattering laws $S(Q, \Delta E)$ of PMMA at $200 \mathrm{~K}\left(T_{g}=377 \mathrm{~K}\right)$ as a function of $Q$ from 1.1 to $4.8 \AA^{-1}$. $S(Q, \Delta E)$ was corrected for the Debye-Waller factor $\exp (-2 W)$. Note that the onset temperature of the fast process is approximately $160 \mathrm{~K}$.

Debye level in the low- $Q$ and low-energy region, and hence we do not observe it due to the lack of the sensitivity of the neutron scattering.

\section{B. Coherent scattering}

Figure 3 shows coherent dynamic scattering laws $S(Q, \Delta E)$ of deuterated PMMA- $d_{8}$ at $200 \mathrm{~K}$ for various $Q$ values from 0.93 to $4.85 \AA^{-1}$, which were corrected for the Debye-Waller factor evaluated from the $Q$ dependence of

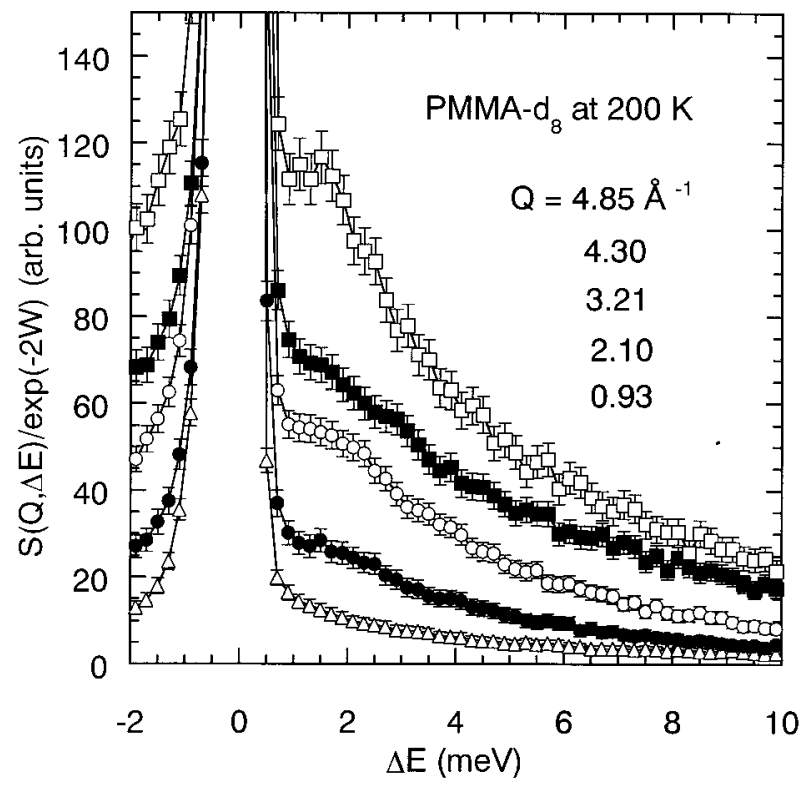

FIG. 3. Coherent dynamic scattering laws $S(Q, \Delta E)$ of PMMA- $d_{8}$ at $200 \mathrm{~K}$ as a function of $Q$ from 0.93 to $4.85 \AA^{-1}$. $S(Q, \Delta E)$ was corrected for the Debye-Waller factor $\exp (-2 W)$.

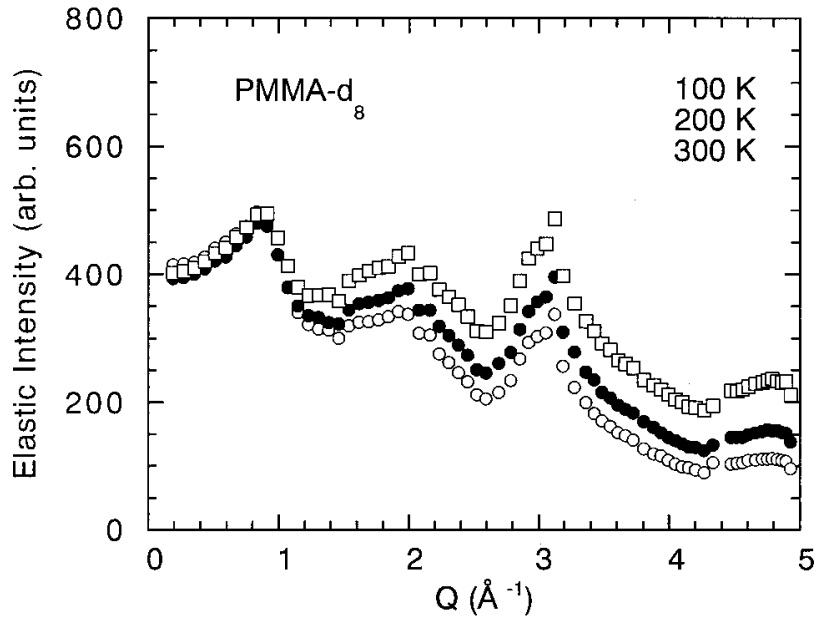

FIG. 4. $Q$ dependence of the coherent elastic scattering from PMMA- $d_{8}$ at $100(\square), 200(\bigcirc)$, and $300(\bigcirc)$ K. Three distinct peaks at $0.9,2.0$, and $3.1 \AA^{-1}$ were observed in the $Q$ range. According to the assignment by Lovell and Windle (Ref. 30), the peak at $0.9 \AA^{-1}$ is a composition containing neighboring interchain correlation and intrachain correlation between nearest-neighboring ester groups in a syndiotactic sequence of atactic PMMA, and the second and third peaks at 2.0 and $3.1 \AA^{-1}$ are intrachain correlations between neighboring ester and methyl groups in a syndiotactic sequence and between nearest-neighboring methylene units in a chain, respectively.

incoherent elastic scattering. Comparing the coherent scattering laws with the incoherent ones shown in Fig. 2, qualitatively no large differences are observed. A similarity between the coherent and incoherent $S(Q, \Delta E)$ is also observed at 100 and $300 \mathrm{~K}$, suggesting that the boson peak and the picosecond-scale process are essentially a self-motion or a very localized motion. One question that we have to consider is to what extent the motions are localized: on one monomer, two monomers, or more?

Information about motional coherency between atoms or atomic groups can be obtained from the $Q$ dependence of coherent inelastic scattering. As is well known, when we observe scattering particles (or atomic groups) moving in phase by coherent scattering, the $Q$ dependence of inelastic scattering due to the in-phase motion must be the same as that of the elastic scattering. On the other hand, if the particles move out of phase, we observe self-motions even in the coherent scattering. On the basis of this simple argument, we discuss the mode of motions related to the boson peak and the picosecond-scale process using the coherent inelastic neutron-scattering data on deuterated PMMA- $d_{8}$.

The $Q$ dependence of elastic scattering intensity is shown in Fig. 4 for 100, 200, and $300 \mathrm{~K}$ in a $Q$ range from 0.19 to $4.93 \AA^{-1}$. Three distinct peaks are observed at $0.9,2.0$, and $3.1 \AA^{-1}$ in the $Q$ range. In a wide-angle x-ray scattering (WAXS) from atactic PMMA, three peaks were observed almost at the same positions: 1.0, 2.2, and $3.0 \AA^{-1}$. The assignment of the three peaks in the WAXS has been done by Lovell and Windle. ${ }^{30}$ This assignment was confirmed by purely coherent neutron scattering using polarized neutrons. ${ }^{22}$ According to them, the first peak at $1.0 \AA^{-1}$ is a 
composition containing neighboring interchain correlations and intrachain correlation between nearest-neighboring ester groups in a syndiotactic sequence of atactic PMMA. The second and third peaks at 2.2 and $3.0 \AA^{-1}$ were assigned to intrachain correlations between neighboring ester and methyl groups in a syndiotactic sequence and between nearestneighboring methylene units in a chain, respectively. Note that interchain correlation is included only in the first peak.

The $Q$ dependences of the coherent inelastic scattering intensities integrated in an energy range from $\Delta E$ $-0.5 \mathrm{meV}$ to $\Delta E+0.5 \mathrm{meV}$ at variable energy transfers $\Delta E$ are shown in Figs. 5(a) -5 (c) for 100, 200, and $300 \mathrm{~K}$, respectively. In the figures, for comparison, the elastic scattering intensities $I_{\mathrm{el}}(Q)$ are also plotted in a form $Q^{2} I_{\mathrm{el}}(Q)$ because inelastic scattering intensity increases with $Q^{2}$. First, we focus on the lowest-energy transfer $\Delta E$ $=1.9 \mathrm{meV}$, which corresponds to the energy region of the boson peak and the picosecond-scale process (see Fig. 1). In the $Q$ dependence of the inelastic scattering at $\Delta E$ $=1.9 \mathrm{meV}$, the peaks or shoulders are observed at 2.0 and $3.2 \AA^{-1}$ at every temperature. These are very close to the peak positions observed in the elastic scattering, although rather broader than those expected from the elastic scattering. On the other hand, no peaks or shoulders are observed at around $Q=0.9 \AA^{-1}$ in the $Q$ dependence of the inelastic scattering intensity at $\Delta E=1.9 \mathrm{meV}$, while a peak at $Q$ $=0.9 \AA^{-1}$ is clearly observed in the expected spectra from the elastic scattering. The absence of the peak at $Q$ $=0.9 \AA^{-1}$ means that at a length scale of $\sim 7 \AA$ $\left(=2 \pi / Q_{\max }\right)$, which includes intrachain and interchain correlations, the boson peak and the picosecond-scale process modes do not move in phase. On the other hand, the peaks at 2.0 and $3.2 \AA^{-1}$ suggesting correlations at a length scale of $\sim 3.1$ and $\sim 2.0 \AA$ are preserved between side chains within a polymer chain. From these observations, it is concluded that the boson peak and the picosecond-scale process modes are intrachain-correlated motion between neighboring side chains and probably localized on two monomers. It is noted that the intrachain-correlated motions are not trivial because there are many rotational degrees of freedom in a PMMA- $d_{8}$ monomer including ester and methyl side chains.

Comparing $S(Q, \Delta E=1.9 \mathrm{meV})$ at $100 \mathrm{~K}$ with those at 200 and $300 \mathrm{~K}$, the peaks at 2.0 and $3.2 \AA^{-1}$ at $100 \mathrm{~K}$ are broader than those at 200 and $300 \mathrm{~K}$. At $100 \mathrm{~K}$, we observe only the boson peak mode, while at $300 \mathrm{~K}$ the picosecondscale process dominants at $\Delta E=1.9 \mathrm{meV}$. The broader peaks at $100 \mathrm{~K}$ probably mean that the boson peak mode has a broader distribution in spatial scale than the picosecond-scale process. As the energy transfer $\Delta E$ increases, intrachain correlation peaks at 2.0 and $3.2 \AA^{-1}$ are less pronounced and not observed at $\Delta E=7.9 \mathrm{meV}$ any longer (see Fig. 5). This suggests that motions in the energy region above $\sim 7 \mathrm{meV}$ are very much localized in a PMMA chain and each monomer and/or side chain moves independently in glassy states.

As mentioned in the Introduction, Buchenau et al. ${ }^{19}$ have performed coherent inelastic scattering measurements on deuterated polybutadiene PB- $d_{4}$, a typical main chain glassforming polymer, focusing on the interchain correlation peak at $Q=1.5 \AA^{-1}$. In the $Q$ dependence of the inelastic scatter-
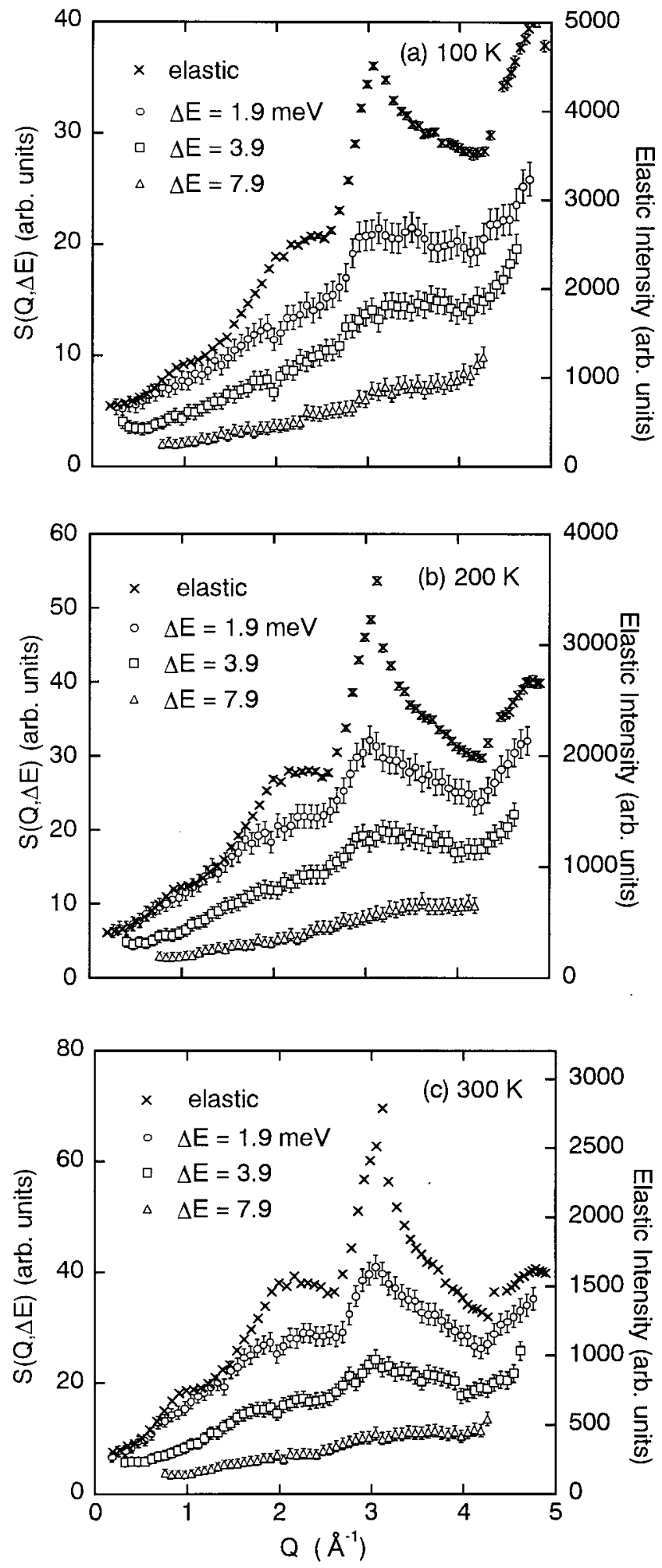

FIG. 5. $Q$ dependence of the coherent inelastic scattering from PMMA- $d_{8}$ as a function of the energy transfer $\Delta E$ from 1.9 to 7.9 meV. (a) $100 \mathrm{~K}$, (b) $200 \mathrm{~K}$, and (c) $300 \mathrm{~K}$. The spectra were obtained by integrating $S(Q, \Delta E)$ in an energy range from $\Delta E-0.5$ to $\Delta E+0.5 \mathrm{meV}$. The $Q$ dependence of the coherent elastic scattering $I_{\mathrm{el}}(Q)$ is also plotted in a form of $Q^{2} I_{\mathrm{el}}(Q)$. 
ing at around $\Delta E=1 \mathrm{meV}$, they observed a clear correlation peak at $Q=1.5 \AA^{-1}$, while no peaks were observed in a high-energy region above $\sim 4 \mathrm{meV}$. Hence they concluded that the picosecond-scale process as well as the boson peak in the low-energy region is a correlated motion between neighboring chains. It was also shown that the low-energy spectra were well reproduced in a model calculation in terms of a sum of sound wave (propagating mode) and localized modes. Their results seem to contradict our observations in PMMA- $d_{8}$. This apparent contradiction between the PMMA- $d_{8}$ and PB- $d_{4}$ results is interpreted in terms of a strong damping of the correlated motion. In PB- $d_{4}\left[-(\mathrm{CD}=\mathrm{CD}-\mathrm{CD}=\mathrm{CD})_{n}-\right]$, which has no side chains, main chains are in close proximity; hence, the correlated motion between the neighboring main chains is not damped. In PMMA- $d_{8}\left[-\left(\mathrm{CD}_{2}-\mathrm{C}\left(\mathrm{CD}_{3}\right)\left(\mathrm{COOCD}_{3}\right)\right)_{n}-\right]$, on the other hand, two side chains, that is $-\mathrm{CD}_{3}$ and $-\mathrm{COOCD}_{3}$, separate the neighboring main chains. In fact, the interchain distance $\left(2 \pi / Q_{\max }\right)$ is $7 \AA$ for PMMA- $d_{8}$, being much larger than that of PB- $d_{4}(4.2 \AA)$. Therefore, the correlated motion between neighboring main chains must be damped due to the existence of side chains, and limited within a polymer chain.

It is worth pointing out that the picosecond-scale process in PMMA and PMMA- $d_{8}$ observed far below $T_{g}$ is not re- lated to the glass transition because it is a side-chain motion, although the apparent features are very similar to those observed in PB except the onset temperature.

\section{CONCLUSIONS}

In the incoherent and coherent inelastic neutron scattering measurements on PMMA and PMMA- $d_{8}$, we have extended the $Q$ range up to $4.9 \AA^{-1}$, keeping a high-energy resolution of $0.16 \mathrm{meV}$. These measurements revealed a localized nature of the picosecond-scale process and the boson peak observed at around $2 \mathrm{meV}$. It was found in the coherent measurements that the boson peak and the picosecond-scale process do not move in phase at a length scale of $\sim 7 \AA$, while the motional coherency is preserved between neighboring side chains within a polymer. From these observations, it was concluded that both of them are intrachaincorrelated motions between neighboring side chains and localized in a length scale less than $\sim 7 \AA$, probably on two monomers. As the excitation energy $\Delta E$ increases, the intrachain motional coherency is less pronounced for both the boson peak and the picosecond-scale process. Above $\sim 7$ $\mathrm{meV}$, this coherency is completely damped, implying that the motions are localized on less than one monomer and the side chains move independently.
*Corresponding Author. FAX: +81-774-38-3146. Electronic address: kanaya@scl.kyoto-u.ac.jp

${ }^{1}$ Dynamics of Glass Transition and Related Topics, edited by T. Odagaki, Y. Hiwatari, and J. Matsui [Suppl. Prog. Theor. Phys. 126 (1997)] and references therein.

${ }^{2}$ Proceedings of The Third International Discussion Meeting on Relaxation in Complex Systems, edited by K. L. Ngai, E. Riande, and M. D. Ingram [J. Non-Cryst. Solids 235/237 (1998)] and references therein.

${ }^{3}$ U. Buchenau, Y. M. Galperin, V. L. Gurevich, and H. R. Schober, Phys. Rev. B 43, 5039 (1991).

${ }^{4}$ H. R. Schober and B. B. Laird, Phys. Rev. B 44, 6746 (1991).

${ }^{5}$ H. R. Schober and C. Oligschleger, Phys. Rev. B 53, 11469 (1996)

${ }^{6}$ F. Sette, M. Krisch, C. Masciovecchio, G. Ruocco, and G. Monaco, Science 280, 1550 (1998).

${ }^{7}$ A. Mermet, C. Cunsolo, D. Vuval, M. Krisch, C. Masciovecchio, S. Perghem, G. Ruocco, F. Sette, R. Verbeni, and G. Viliani, Phys. Rev. Lett. 80, 4205 (1998).

${ }^{8}$ U. Buchenau, C. Pecharroman, R. Zorn, and B. Frick, Phys. Rev. Lett. 77, 659 (1996)

${ }^{9}$ T. Kanaya, I. Tsukushi, K. Kaji, B. Gabrys, and S. M. Bennington, J. Non-Cryst. Solids 235/237, 212 (1998).

${ }^{10}$ T. Kanaya, U. Buchenau, S. Koizumi, I. Tsukushi, and K. Kaji, Phys. Rev. B 61, R6451 (2000).

${ }^{11}$ B. Frick, D. Richter, W. Petry, and U. Buchenau, Z. Phys. B: Condens. Matter 70, 73 (1988).

${ }^{12}$ T. Kanaya, T. Kawaguchi, and K. Kaji, J. Chem. Phys. 98, 8262 (1993).

${ }^{13}$ T. Kanaya, T. Kawaguchi, and K. Kaji, J. Chem. Phys. 105, 4342 (1996)
${ }^{14}$ B. Frick, U. Buchenau, and D. Richter, Colloid Polym. Sci. 273, 421 (1995).

${ }^{15}$ T. Kanaya, T. Kawaguchi, and K. Kaji, J. Chem. Phys. 104, 3841 (1996).

${ }^{16}$ J. Hansen, T. Kanaya, K. Nishida, K. Kaji, K. Tanaka, and A. Yamaguchi, J. Chem. Phys. 108, 6492 (1998).

${ }^{17}$ F. Fujara and W. Petry, Europhys. Lett. 4, 921 (1987).

${ }^{18}$ B. Frick and D. Richter, Phys. Rev. B 47, 14795 (1993).

${ }^{19}$ U. Buchenau, A. Wischnewski, and D. Richter, Phys. Rev. Lett. 77, 4035 (1996).

${ }^{20}$ G. E. Bacon, Neutron Diffraction (Clarendon, Oxford, 1975).

${ }^{21}$ B. Gabrys, J. S. Higgins, and O. Schärpf, J. Chem. Soc., Faraday Trans. 1 82, 1923 (1986).

${ }^{22}$ B. Gabrys, J. S. Higgins, and O. Schärpf, J. Chem. Soc., Faraday Trans. 1 82, 1929 (1986).

${ }^{23}$ M. Bee, Quasielastic Neutron Scattering: Principles and Applications in Solid State Chemistry, Biology and Materials Science (Hilger, Bristol, 1988).

${ }^{24}$ U. Buchenau, M. Prager, N. Nücker, A. J. Dianoux, N. Ahmad, and W. A. Phillips, Phys. Rev. B 34, 5665 (1986).

${ }^{25}$ K. Inoue, T. Kanaya, S. Ikeda, K. Kaji, K. Shibata, M. Misawa, and Y. Kiyanagi, J. Chem. Phys. 95, 5332 (1991).

${ }^{26}$ O. Yamamuro, T. Matsuo, K. Takeda, T. Matsuo, T. Kanaya, T. Kawaguchi, and K. Kaji, J. Chem. Phys. 105, 732 (1996).

${ }^{27}$ B. Frick, D. Richter, R. Zorn, and L. J. Fetters, J. Non-Cryst. Solids 172/174, 272 (1994).

${ }^{28}$ A. Mermet, E. Duval, N. V. Surovtsev, J. F. Jal, A. J. Dianoux, and A. F. Yee, Europhys. Lett. 38, 515 (1997).

${ }^{29}$ B. Frick, D. Richter, R. Zorn, and L. J. Fetters, J. Non-Cryst. Solids 172/174, 272 (1994).

${ }^{30}$ R. Lovell and A. H. Windle, Polymer 22, 175 (1981). 